\title{
Eukaryotic nucleotide excision repair: from understand- ing mechanisms to influencing biology
}

\author{
Sarah C Shuck ${ }^{1}$, Emily A Short ${ }^{2}$, John J Turchi ${ }^{1,2}$ \\ ${ }^{I}$ Department of Biochemistry and Molecular Biology, Indiana University School of Medicine, Indianapolis, IN 46202, USA; ${ }^{2}$ Division \\ of Hematology and Oncology, Indiana University School of Medicine, Indianapolis, IN 46202, USA
}

Repair of bulky DNA adducts by the nucleotide excision repair (NER) pathway is one of the more versatile DNA repair pathways for the removal of DNA lesions. There are two subsets of the NER pathway, global genomic-NER (GGNER) and transcription-coupled NER (TC-NER), which differ only in the step involving recognition of the DNA lesion. Following recognition of the damage, the sub-pathways then converge for the incision/excision steps and subsequent gap filling and ligation steps. This review will focus on the GGR sub-pathway of NER, while the TCR sub-pathway will be covered in another article in this issue. The ability of the NER pathway to repair a wide array of adducts stems, in part, from the mechanisms involved in the initial recognition step of the damaged DNA and results in NER impacting an equally wide array of human physiological responses and events. In this review, the impact of NER on carcinogenesis, neurological function, sensitivity to environmental factors and sensitivity to cancer therapeutics will be discussed. The knowledge generated in our understanding of the NER pathway over the past 40 years has resulted from advances in the fields of animal model systems, mammalian genetics and in vitro biochemistry, as well as from reconstitution studies and structural analyses of the proteins and enzymes that participate in this pathway. Each of these avenues of research has contributed significantly to our understanding of how the NER pathway works and how alterations in NER activity, both positive and negative, influence human biology.

Keywords: nucleotide excision repair, xeroderma pigmentosum, DNA damage recognition, global genomic NER Cell Research (2008) 18:64-72. doi: 10.1038/cr.2008.2; published online 1 January 2008

\section{Xeroderma pigmentosum (XP): a genetic disease that defines NER}

Xeroderma pigmentosum (XP), an autosomal recessive disease with 7 complementation groups and a single variant, is categorized by extreme sensitivity to sunlight and predisposition to cancer. The clinical manifestations of this disease stem from a decrease in DNA repair caused by mutations in proteins of the nucleotide excision repair (NER) pathway. In 1968, a direct link was found between DNA repair and carcinogenesis following the observation that cells derived from XP patients were unable to repair ultraviolet (UV)-induced DNA damage, leading to an increased predisposition to cancer [1]. Since that time, the connection between DNA repair and cancer has been rediscovered nu-

Correspondence: John J Turchi

Indiana University Cancer Research Institute, 1044 W. Walnut, R4-202, Indianapolis, IN 46202, USA

Tel: +1 317278 1996; Fax: +1 3172740396

E-mail: jturchi@iupui.edu merous times. The analysis of XP allowed the delineation of the NER pathway with each complementation group, XPA through XPG, corresponding to an essential protein in the pathway (Table 1). Each protein in this pathway has been studied in great detail with respect to activity, interactions and post-translational modifications (PTMs). A subset of the XP proteins participate in other pathways including the repair of oxidative damage, removal of DNA cross-links and transcription $[2,3]$. The dual role of these proteins at the molecular level explains other manifestations of XP including neurological symptoms. Recent analysis of XP genetics has focused on variations in expression level and single-nucleotide polymorphisms (SNPs) as they relate to a variety of physiological responses, including susceptibility to certain cancer types, response to DNA damaging chemotherapeutic agents and aging. The convergence of genetic and biochemical analyses will ultimately allow the determination of the impact of NER SNPs on DNA repair capacity, which is the true measure of the biological and physiological effects of SNPs. Much of the basic enzymology of NER is evolutionarily conserved from prokaryotes, 
Table 1 NER factors

\begin{tabular}{|c|c|c|c|}
\hline Factor & Subunits/associations & Activity & PTM \\
\hline XPA & p32 & Damage verification & Phosphorylation \\
\hline XPB & TFIIH & Helicase & \\
\hline XPC & Centrin-2 & & Sumoylation \\
\hline XPD & TFIIH & Helicase & \\
\hline $\mathrm{XPF}$ & ERCC1 & Incision & \\
\hline XPG & & Incision & \\
\hline \multirow[t]{2}{*}{ RPA } & $\mathrm{p} 70 / \mathrm{p} 34 / \mathrm{p} 14$ & Damage recognition & Phosphorylation \\
\hline & & Resynthesis & \\
\hline
\end{tabular}

including damage recognition, dual incision and repair synthesis. These findings are highlighted in a recent, excellent review on prokaryotic NER [4]. Analysis of NER in both eukaryotic and prokaryotic systems has elucidated the biochemical mechanisms of XP. Over the past decade there have been major strides in our understanding of XP and its relationship to DNA repair, thus advancing our knowledge of NER. Such work has allowed us to more broadly determine how variations in NER proteins, including expression level, mutations and SNPs, can increase an individual's susceptibility to cancer as well as predict the response to chemotherapeutic treatments.

\section{In vitro reconstitution of NER}

The NER pathway removes bulky DNA adducts caused by UV irradiation and chemical mutagens. The repair of DNA damage begins with a damage recognition step and assembly of a pre-incision complex, followed by excision of the damaged strand and gap-filling DNA synthesis. The establishment of a quantitative, reliable in vitro NER activity assay has resulted in the purification and characterization of the individual proteins and complexes via biochemical complementation of XP cell-free extracts [5] (Table 1).

The six core factors that have been implicated in the damage recognition and dual incision steps of global genomic-NER (GG-NER) are the XPC-RAD23B complex, transcription factor IIH (TFIIH), XPA, replication protein A(RPA), XPG, and XPF-ERCC1 [6]. In addition, the damaged DNA-binding protein (DDB), a heterodimer of $\mathrm{p} 120$ and $\mathrm{p} 48$ subunits, plays a role in the recognition process of certain adducts [7]. While initial work indicated that the p120 subunit was linked to XPE, subsequent analysis revealed that in all bona fide XPE cells the defect mapped to the DDB2 gene which encodes the $\mathrm{p} 48$ subunit [8]. It is generally accepted that XPA, RPA, TFIIH and XPCRAD23B comprise the initial recognition, or pre-incision, complex. XPA, a $40 \mathrm{kDa}$ zinc metalloprotein, is an integral protein in NER and is unique in the sense that, unlike most NER factors, it does not appear to play a role in other biochemical processes in the cell. XPA interacts with many of the core repair factors in NER and, without XPA, no stable pre-incision complex can form, nor can NER occur $[9,10]$. In addition, XPA has recently been demonstrated as the limiting factor in NER damage recognition [11]. RPA, a heterotrimeric protein composed of 70,32 and 14 $\mathrm{kDa}$ subunits, is a single-stranded DNA binding protein that is involved in several cellular processes, including NER, recombination, DNA replication and signaling by DNA damage checkpoints [12]. RPA plays an integral role in recognition preceding incision and in post-excision repair synthesis. XPC is a $106 \mathrm{kDa}$ protein that forms a stable complex with RAD23B, which is a $43 \mathrm{kDa}$ human homolog of the yeast NER protein RAD23 [13]. Centrin 2 has been shown to interact with XPC-RAD23B and, while this component is not required for in vitro NER incision of UV-damaged DNA repair is stimulated by centrin 2 [14]. In vivo relevance of this interaction was demonstrated by studying a XPC mutant that is unable to bind centrin 2 , which abrogated the stimulatory effect of centrin-2 on incision activity in vitro, and rendered cells hypersensitive to UV irradiation [14]. Of all the proteins involved in NER, XPC has the strongest affinity for both damaged and undamaged DNA [15]. Centrin-2 was also found to stimulate both the damaged and undamaged DNA-binding activity of XPC [14].

XPC-RAD23B has a critical role in the recruitment 
of TFIIH and interacts strongly with this complex [16]. TFIIH is a nine-subunit complex that functions in both NER and transcription initiation. Two of its subunits, XPB and XPD, are DNA helicases which unwind the damaged duplex DNA [17]. Once the DNA has been unwound, XPG and XPF-ERCC1 are sequentially employed to make the $3^{\prime}$ and $5^{\prime}$ incisions, respectively, on each side of the lesion [10]. Following incision, the damage-containing oligomer of approximately 24 to 32 nucleotides is excised and the DNA structure is restored via the gap-filling stage of NER, which requires replication factor C (RFC), PCNA, DNA polymerase $\delta$ or $\varepsilon$, DNA ligase I and RPA [18]. Very recent results have demonstrated that the gap-filling and ligation step in NER may also be accomplished by an alternative pathway. In a DNA polymerase $\delta$-dependent reaction, XRCC 1 and DNA ligase III $\alpha$ have been demonstrated to be required for gap filling in quiescent cells [19]. The authors also demonstrated a cell cycle dependence whereby the DNA ligase I pathway is only active in late G1/S cells. These recent advances provide considerable insight into what was typically thought to be a relatively unregulated step in NER and highlight the importance of continued investigation into the basic mechanisms of DNA repair.

\section{Recognition of DNA damage by NER factors}

In order for the NER pathway to repair bulky DNA adducts, the factors involved must be able to distinguish DNA damage sites from the vast background of undamaged DNA. Unlike TC-NER, GG-NER is initiated not by the stalling of a DNA metabolic protein but rather by specific recognition of the DNA damage site by proteins in the NER pathway. NER proteins are able to discern damaged DNA from the undamaged DNA due to the ability of certain proteins in the pathway to identify areas of bulky DNA lesions that have undergone helical distortions.

The three factors involved in the recognition step of GGR, XPC-RAD23B, XPA and RPA, all show a binding preference to damaged duplex DNA compared to undamaged. Numerous models have been proposed to illustrate the order of assembly of proteins at the site of DNA damage and the mechanism of the NER pathway. One of the first theories held that a preassembled complex, with all six core factors, was able to carry out the entire excision reaction. Several lines of investigation, however, argue against the existence of a cellular preformed complex capable of carrying out all the necessary steps in NER (reviewed in ref. [20]).

The precise order of assembly of these core recognition factors on the site of DNA damage has been the subject of many studies, with early in vitro data supporting models of either XPC-RAD23B or XPA-RPA as the first complex that binds to a UV-induced DNA lesion [21, 22]. While initial evidence suggests that RPA or an RPA-XPA complex binds first to the damage and subsequently recruits XPC-RAD23B with TFIIH, more recent analyses support the hypothesis that XPC-RAD23B is the primary damage recognition factor [23-25]. A likely cause for inconsistent findings in regard to the order of assembly can be attributed either to the variety of experimental assays and conditions employed, or to the fact that no specific order is in fact necessary.

Given the absence of a preformed complex, the assembly of NER factors at the site of a UV-induced DNA lesion must occur in either a sequential, ordered process or a random addition process. The term "recruitment" is often used to describe the assembly of protein factors in a variety of cellular pathways including NER. The idea of "recruiting" a protein to a site of DNA damage or the assembly of factors to form an incision nuclease, while satisfying, is a descriptive observation. The mechanisms of "recruitment" have, however, yet to be discerned. In the absence of a yet-tobe-discovered soluble signaling molecule emanating from the site of damage in a chemotactic-like model, assembly at the sites of DNA damage in the GGR pathway of NER must include components for the kinetics of association and dissociation and the local concentrations of relevant proteins. Our understanding of the assembly process has been greatly aided more recently by cell-based studies of repair rates and measurement of assembly at the sites of damage. Immunofluorescence analysis and the use of fluorescent tagged NER factors have enabled a sub-set of interactions to be better characterized. For example, analyses revealed that XPC-RAD23B is required for TFIIH assembly at the sites of DNA damage [23]. These results and analysis of various XP cell lines led to the conclusion that XPC-RAD23B represents the first factor bound at the sites of DNA damage. Analysis of the XPA dynamics revealed that XPA rapidly accumulates at the sites of DNA damage in an XPC-dependent manner [26]. These results are consistent with but do not prove the hypothesis that XPC represents the first molecule bound at the site of a UVinduced DNA lesion. For example, transient XPA binding to a UV-damage site with a fast rate of dissociation would result in the inability to localize XPA at the lesion in the absence of XPC. If XPC-RAD23B decreased the rate of XPA dissociation, the accumulation of XPA at the sites of UV damage would then only be observed in XPC-positive cells. A second consideration in the studies of NER protein dynamics in XP cell lines is whether proteins which are not required for a specific sub-pathway, TCR or GGR, participate in that pathway when such proteins are present in the cell. For instance, even though XPC is not required for TCR, in XPC wild-type cells, does the XPC-TFIIH 
interaction result in XPC localization with stalled RNA polymerase II at UV damage sites? Additional studies of NER in live cells will undoubtedly allow these interesting questions to be answered to better define the cellular response to UV damage.

More recently, mathematical modeling has been employed in attempts to elucidate the order of addition of NER factors at the sites of UV damage. Similar to the in vitro and cellular repair data, the models yield disparate results. Using a model without including RPA as a recognition factor indicates that a sequential ordered process results in the most efficient excision [27]. More recent modeling however favors a random order-cooperative binding/kinetic proofreading model [28]. This model incorporates RPA as a recognition factor and includes potential dissociation of NER factors, and supports RPA, XPA and XPC-RAD23B assembly at the site of damage. This model also invokes a cooperativity function in binding to achieve a higher specificity for damaged DNA. This specificity is enhanced by the kinetic proofreading activity of TFIIH to verify the recognition step [28]. This represents an interesting treatment of the damage recognition step in NER and highlights the importance of the kinetics of the interactions. Evidence is also presented suggesting that random assembly provides kinetic advantages to a sequential model of assembly [28]. While considerable evidence suggests that XPC-RAD23B is the main initiator of GGR, how well the model reflects the damage recognition process in vivo in the vast majority of cells where all the factors are present remains to be determined.

Once a site of damage is identified, DNA excision occurs exclusively on the DNA strand that contains the DNA adduct. This indicates that the proteins involved in NER are able to distinguish not only damaged vs. undamaged duplex DNA but also which strand contains the adduct [29]. Pre-steady-state kinetic analysis of both RPA and XPCRAD23B demonstrates that both of these factors display an increased rate of dissociation from damaged singlestranded DNA compared to undamaged single-stranded DNA $[30,31]$. These results suggest the involvement of both factors in the discrimination of the damaged DNA strand versus undamaged DNA strand of the duplex DNA. The finding that each of these factors contains this potential discriminatory activity suggests that this represents an early step in the recognition process, and that the strand to be incised is potentially determined prior to the addition of XPB and XPD helicases in the TFIIH complex. The model presented in Figure 1 highlights the structural changes in the DNA duplex accompanying binding of the individual NER factors. The potential for reversible binding of multiple factors is highlighted in the first reversible step with a committed, irreversible second step. If the initial binding were to occur at an undamaged site, the second step would also be reversible to limit incision at undamaged sites.

\section{NER protein-induced DNA structural changes}

XPC-RAD23B has been implicated as the first protein in

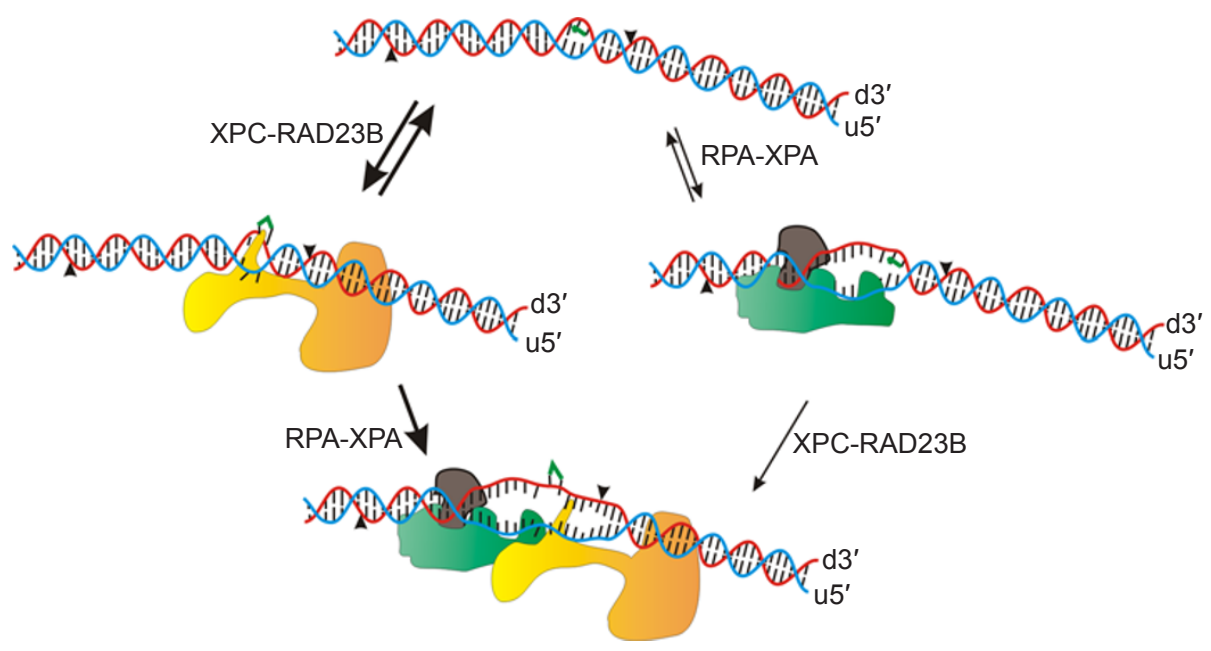

Figure 1 Recognition of bulky adduct-DNA damage. Bulky DNA adducts distort DNA structure and the initial binding by XPCRAD23B is potentially accompanied by insertion of a $\beta$-hairpin between the strands of the duplex. Similarly if the RPA-XPA complex encounters a damaged DNA, localized unwinding occurs inducing a greater distortion in the duplex. These potential pathways converge with the addition of the second protein complex recognizing the greater distortion of the duplex. The position of endonucleolytic cleavage is indicated by the carats and the position of the DNA adduct is depicted in green on the damaged strand. The damaged strand is denoted with the "d" and the undamaged strand with the "u". The orientation is denoted for each strand of the duplex. 
this pathway to interact with DNA and has been shown to induce the first structural changes in the DNA required for NER, specifically the opening of the DNA double helix [10, 32]. XPC-RAD23B has been shown in vitro to bind better to duplex DNA containing helix-distorting lesions, such as cisplatin adducts, compared to undamaged DNA [31,33]. However, when comparing the ability of XPC-RAD23B to bind to DNA containing less helix-distorting lesions, such as cyclobutane pyrimidine dimers (CPDs), with undamaged DNA, no difference in binding is observed [34]. This increased recognition of more helix-distorting lesions has also been observed in examination of $1,3 \mathrm{GpXpG}$ vs. 1,2 GpG cisplatin-DNA adducts. XPC-RAD23B is better able to recognize the more helix-distorting $1,3 \mathrm{GpXpG}$ cisplatin adducts compared to the 1,2 lesions [31]. An interesting observation has been made in which XPC-RAD23B displays decreased binding affinity for less helix-distorting lesions but is still able to discriminate between damaged and undamaged DNA substrates as only the damaged duplex DNA undergoes repair [34]. The very recent structural analysis of yeast XPC ortholog, $\operatorname{Rad} 4 / \operatorname{Rad} 23$, with a CPD damaged DNA confirms and extends many of these findings [35]. The structure reveals the insertion of a beta-strand between the DNA strand of the duplex with concomitant "flipping out" of the bases opposite the lesion. A similar model was also observed for the uvrB protein involved in prokaryotic NER [36]. The "flipping out" of bases via insertion of beta sheets represents a common theme for DNA repair proteins [37-41] and leads to a series of interesting questions regarding how mammalian NER proteins assemble on damaged DNA. Do the extra helical bases increase the affinity of RPA and XPA for the structure or does the presence of RPA-XPA increase XPC-dependent changes in the structure of the DNA? These types of dynamic interactions necessitate the use of kinetic analysis to assess how the interaction influences both DNA structure and assembly of proteins as intermediates in the DNA damage recognition process.

XPA, like XPC-RAD23B, is required for the opening of the DNA duplex, but is further downstream from the lesion than XPC-RAD23B [42]. Solution structure work has shown that XPA is able to interact directly with DNA via its zinc-containing domain [43]. Previous work has shown that the ability of XPA to bind damaged DNA increases as deformation of the DNA increases [44]. Further work demonstrated that two conserved lysines, 141 and 179, are important for damage recognition and that kinking of the DNA in regions of damage increases electrostatic potential, which aids in XPA recognition of the damaged DNA [45]. XPA has also been demonstrated to contact both strands of the duplex DNA and inhibit RPA-dependent DNA unwinding $[46,47]$. These results suggest that XPA may stabilize the single-strand/double-strand DNA junction $5^{\prime}$ of the damage (Figure 1).

RPA, which binds ssDNA with a high affinity using six oligonucleotide/oligosaccharide (OB) folds, enhances the ability of XPA to recognize damaged DNA [47]. Like the other proteins involved in the DNA damage recognition step of NER, RPA binds damaged duplex DNA via a kinetic mechanism involving a higher rate of association and lower rate of dissociation when comparing damaged versus undamaged duplex DNA [30, 48]. Although RPA binds damaged duplex DNA better than undamaged, it still binds ssDNA with a greater affinity than either type of duplex [47]. Its ability to bind damaged DNA may come from the distortion produced by bulky adducts as well as the DNA unwinding that other proteins in the pathway induce. The nature of the adduct itself or binding XPC-RAD23B produces a substrate that has single-stranded character to which RPA can recognize and bind. RPA has been shown to unwind duplex linear DNA [49] and binding of RPA to cisplatin-damaged DNA generates single-stranded DNA [50]. When XPA forms an RPA-XPA-binding complex, the affinity for damaged DNA and the stability of the damaged DNA duplex increases [47]. This is evidenced by a decrease in the amount of single-stranded DNA that is generated by the binding of RPA to duplex damaged DNA [47]. Although the amount of single-stranded DNA that is generated is decreased, kinetic analysis examining binding of XPA and RPA to duplex damaged DNA indicates a biphasic reaction that is consistent with denaturation of the duplex DNA and binding of RPA to the single-stranded DNA [47]. Therefore, RPA's role in NER may be in the recognition of the singlestranded DNA formed by the preincision complex.

\section{Modulation of NER via PTMs and protein-protein interactions}

The ability of proteins to participate in the NER pathway is regulated by several factors including PTM and interactions with other proteins. XPC-RAD23B has been shown to be ubiquitylated in response to UV light by the DDB2-E3 ubiquitin ligase complex, a modification shown to be required for cell-free NER of UV-induced 6-4 photoproducts [51]. SUMO-1 modification of XPC has also been reported in response to UV-induced DNA damage [52]. While the definitive role of SUMO-1 modification has not been determined, the potential exists that this modification negatively impacts ubiquitylation. More recent studies have revealed considerable insights into the association of DDB2 and XPC with UV-damaged DNA [53]. DDB2 was found to associate with and dissociate from UV-damage sites independent of XPC. DDB2 degradation following UV damage was also uncoupled from (UV-damaged) DNA 
binding. A model for DDB2 regulation of chromatin was proposed, whereby accessibility of NER complexes is impacted by preassociation with DDB [53].

The PTM of XPA has also been recently reported. Phosphorylation at XPA serine 196 has been shown to be required for cell survival in response to UV treatment, but no analysis of NER activity was performed. Thus, the direct role or importance of this residue in assembly and disassembly of NER complexes is unclear [54]. RPA is also regulated by phosphorylation; however, it does not seem to have an effect on NER activity [55]. Although phosphorylation does not have an effect on NER activity, RPA is hyperphosphorylated in response to UV treatment and hydroxyurea, and this phosphorylation results in slightly reduced binding to duplex damaged and undamaged DNA $[56,57]$. In the background of overall lower DNA-binding activity, hyperphosphorylated RPA still retains specificity for cisplatin-damaged DNA versus undamaged DNA [56, 57]. The major impact of RPA hyperphosphorylation is on protein-protein interactions. Hyperphosphorylation of RPA resulted in reduced binding to DNA polymerase $\alpha$, ATM and DNA-PK, while having no effect on XPA binding [56, 58]. To ultimately determine the mechanisms by which these PTMs influence protein-protein interactions, detailed analysis of the points of contact needs to be performed. Preferably, these interactions must be studied in the context of full-length proteins and higher-ordered complexes. Studies of this type will undoubtedly benefit from technological advances, especially in analytical techniques, including protein modification and mass spectrometry, which can be used to identify precise sites of interaction.

\section{NER polymorphisms and cancer predisposition}

Considering the long history of associating NER and carcinogenesis, NER genes have been the subject of intense scrutiny using advances in the analysis of SNPs [59-64]. The hypothesis that decreased DNA repair capacity results in increased mutation rates and genetic instability that eventually increase cancer development is firmly supported by a variety of rare human diseases including hereditary nonpolyposis colorectal cancer (HNPCC), XP and ataxia telangiectasia (AT). Extending this hypothesis to the general population to ascertain cancer risk has been driven by technological advances in the analysis of human gene expression and polymorphisms. Many of these analyses are contradictory and present a considerable challenge in delineating true connections and relationships. An additional shortcoming of many of these studies is that they often do not measure the parameter on which the above hypothesis is based, DNA repair capacity. Results obtained analyzing XPD SNPs highlight this disconnect between genetic marker analysis and biochemical ramification and was the subject of a recent review [65]. The three most common XPD SNPs include R156R, D312N and K751Q. Contradictory results have been obtained in the analysis of these SNPs in nearly every cancer type tested. More recent meta-analysis of the entire series of studies revealed that the $\mathrm{R} 156 \mathrm{R}$, which is a synonymous polymorphism, is important in skin cancer [66]. The mechanism by which this genetic change could impact DNA repair is unclear unless this SNP is associated with another SNP that directly impacts DNA repair. The authors also identified significant associations between the 312 and 751 SNPs and skin, breast and lung cancers [66]. Despite neither amino acid being highly conserved or in a region of the protein thought to impact function, UV sensitivity of peripheral blood lymphocytes (PBLs) from individuals with $312 \mathrm{~N}$ and 751Q was greater than that from those with 312D and 751K [67], consistent with decreased NER capacity. The analysis of an XPC SNP K939Q has uncovered a correlation with certain cancers [68-71]. This SNP maps to a critical region of XPC, the extreme C-terminal domain, that is crucial for interaction with TFIIH [72], thus providing a potential biochemical basis for decreased DNA repair capacity and increased cancer risk. Despite the limitations of many of these studies, the analysis of DNA repair SNPs does reveal potentially interesting associations. Further analyses at the genetic and biochemical level are needed to determine the true impact of these individual SNPs. While many studies have reported on a subset of DNA repair factor polymorphisms, a recent study evaluated a wide array of DNA repair factor SNPs and reported that individual SNPs offer little predictive power. Recent analyses of multiple SNPs within the DNA repair pathway have resulted in greater predictive capacity for cancer risk and response to DNA-damaging chemotherapeutics $[73,74]$.

\section{NER and response to chemotherapeutics}

In addition to a role in carcinogenesis, NER capacity is also relevant in the context of cancer treatment. Numerous chemotherapeutic agents, including cisplatin, act via the formation of bulky DNA adducts. Increased DNA repair capacity would be anticipated as detrimental to cisplatin efficacy, while decreased DNA repair capacity would result in a more positive initial response to the therapy. Cisplatin is a front-line treatment for a variety of cancers, including testicular, lung and ovarian cancers, as well as tumors of the head and neck [75-79]. The importance of DNA repair capacity in influencing chemosensitivity has been born out in a subset of cancers and treatments. The most clear-cut case is most likely that of testicular cancer, where cisplatin-based treatment regimens achieve a 95\% cure rate [80]. This phe- 
nomenal clinical outcome is correlated with dramatically reduced DNA repair capacity in testicular cancer cells as a result of decreased levels of the critical XPA protein [11, 81, 82]. Other proteins involved in the NER pathway, including ERCC1 and XPF, have also been shown to be reduced in testis tumor cell lines [82]. Conversely, patients with nonsmall cell lung cancer (NSCLC) present with higher levels of DNA repair capacity and an inverse correlation has been made between survival rate and DNA repair capacity [83]. Considering these activities and correlations, targeting the NER pathway has the potential to increase the efficacy of current chemotherapeutic agents that work by damaging DNA [84]. In particular, the RPA-XPA complex is one target currently under investigation, and small-molecule inhibitors (SMIs) of RPA have been identified via in vitro high-throughput screening [85]. We have extended these studies to include identification of SMIs that prevent XPA binding to DNA. The ability to prevent XPA binding to DNA using SMIs will stop the NER pathway at the recognition step and prevent downstream signals for repair. In contrast, targeting RPA with an SMI has the potential to perturb several DNA metabolic pathways, including DNA replication, recombination and repair. While the search for inhibitors with increased specificity drives much of current drug discovery efforts, recent analysis of kinase inhibitors indicates that broad inhibition of important classes of targets can yield dramatic clinical responses.

\section{Conclusions}

Building on the foundation established by early studies of XP and advances in our understanding of the mechanics of the repair process and how it is regulated, the field of NER has re-emerged as a key component in the research of carcinogenesis, cancer treatment and, more recently, aging $[86,87]$. With the current focus on translating this knowledge towards benefiting human health, the challenge will be to maintain the generation of new knowledge in this field and to expand the foundation of the basic mechanics of NER and how the NER pathway impacts human biology.

\section{Acknowledgments}

This work was supported by NIH award CA82741 and additional funding from the Flight Attendants Medical Research Institute and the Walther Cancer Institute.

\section{References}

1 Cleaver J. Defective repair replication of DNA in xeroderma pigmentosum. Nature 1968; 218:652-656.

2 D'Errico M, Parlanti E, Teson M, et al. New functions of XPC in the protection of human skin cells from oxidative damage.
EMBO J 2006; 25:4305-4315.

3 Niedernhofer LJ, Odijk H, Budzowska M, et al. The structurespecific endonuclease Ercc1-Xpf is required to resolve DNA interstrand cross-link-induced double-strand breaks. Mol Cell Biol 2004; 24:5776-5787.

4 Truglio JJ, Croteau DL, Van Houten B, Kisker, C. Prokaryotic nucleotide excision repair: the UvrABC system. Chem Rev 2006; 106:233-252.

5 Wood RD, Robins P, Lindahl T. Complementation of the xeroderma pigmentosum DNA repair defect in cell-free extracts. Cell 1988; 53:97-106.

$6 \mathrm{Mu}$ D, Park CH, Matsunaga T, Hsu DS, Reardon JT, Sancar A. Reconstitution of human DNA repair excision nuclease in a highly defined system. J Biol Chem 1995; 270:2415-2418.

7 Wakasugi M, Shimizu M, Morioka H, Linn S, Nikaido O, Matsunaga, T. Damaged DNA-binding protein DDB stimulates the excision of cyclobutane pyrimidine dimers in vitro in concert with XPA and replication protein A. J Biol Chem 2001; 276:1543415440 .

8 Itoh T, Linn S, Ono T, Yamaizumi M. Reinvestigation of the classification of five cell strains of xeroderma pigmentosum group $\mathrm{E}$ with reclassification of three of them. Journal of Investigative Dermatology 2000; 114:1022-1029.

9 Mu D, Wakasugi M, Hsu D, Sancar, A. Characterization of reaction intermediates of human excision repair nuclease. $J$ Biol Chem 1997; 272:28971-28979.

10 Evans E, Moggs JG, Hwang JR, Egly J, Wood, RD. Mechanism of open complex and dual incision formation by human nucleotide excision repair factors. EMBO J 1997; 35:2157-2167.

11 Koberle B, Roginskaya V, Wood RD. XPA protein as a limiting factor for nucleotide excision repair and UV sensitivity in human cells. DNA Repair (Amst) 2006; 5:641-648.

12 Wold MS. Replication protein A: a heterotrimeric, single-stranded DNA- binding protein required for eukaryotic DNA metabolism. Annual Review of Biochemistry 1997; 66:61-92.

13 Thoma BS Vasquez KM. Critical DNA damage recognition functions of XPC-RAD23B and XPA-RPA in nucleotide excision repair. Mol Carcinog 2003; 38:1-13.

14 Nishi R, Okuda Y, Watanabe E, et al. Centrin 2 stimulates nucleotide excision repair by interacting with xeroderma pigmentosum group C protein. Mol Cell Biol 2005; 25:5664-5674.

15 Wakasugi M Sancar A. Order of assembly of human DNA repair excision nuclease. J Biol Chem 1999; 274:18759-18768.

16 Araujo SJ, Nigg EA, Wood RD. Strong functional interactions of TFIIH with XPC and XPG in human DNA nucleotide excision repair, without a preassembled repairosome. Mol Cell Biol 2001; 21:2281-2291.

17 Araujo SJ, Tirode F, Coin F, et al. Nucleotide excision repair of DNA with recombinant human proteins: definition of the minimal set of factors, active forms of TFIIH, and modulation by CAK. Genes Dev 2000; 14:349-359.

18 Shivji MK, Podust VN, Hubscher U, Wood RD. Nucleotide excision repair DNA synthesis by DNA polymerase epsilon in the presence of PCNA, RFC, and RPA. Biochemistry 1995; 34:5011-5017.

19 Moser J, Kool H, Giakzidis I, Caldecott K, Mullenders LH, Fousteri MI. Sealing of chromosomal DNA nicks during nucleotide excision repair requires XRCC1 and DNA ligase III alpha in a cell-cycle-specific manner. Mol Cell 2007; 27:311-323.

20 Park CJ Choi BS. The protein shuffle. Sequential interactions 
among components of the human nucleotide excision repair pathway. FEBS J 2006; 273:1600-1608.

21 Sugasawa K, Ng JMY, Masutani C, et al. Xeroderma pigmentosum group $\mathrm{C}$ protein complex is the initiator of global genome nucleotide excision repair. Mol Cell 1998; 2:223-232.

22 Wakasugi M, Sancar A. Order of assembly of human DNA repair excision nuclease. J Biol Chem 1999; 274:18759-18768.

23 Volker M, Mone MJ, Karmakar P, et al. Sequential assembly of the nucleotide excision repair factors in vivo. Mol Cell 2001; 8:213-224.

24 Sugasawa K, Ng JM, Masutani C, et al. Xeroderma pigmentosum group $\mathrm{C}$ protein complex is the initiator of global genome nucleotide excision repair. Mol Cell 1998; 2:223-232.

25 Riedl T, Hanaoka F, Egly JM. The comings and goings of nucleotide excision repair factors on damaged DNA. EMBO J 2003; 22:5293-5303.

26 Rademakers S, Volker M, Hoogstraten D, et al. Xeroderma pigmentosum group A protein loads as a separate factor onto DNA lesions. Mol Cell Biol 2003; 23:5755-5767.

27 Politi A, Mone MJ, Houtsmuller, AB, et al. Mathematical modeling of nucleotide excision repair reveals efficiency of sequential assembly strategies. Mol Cell 2005; 19:679-690.

28 Kesseler KJ, Kaufmann WK, Reardon JT, Elston TC, Sancar A. A mathematical model for human nucleotide excision repair: damage recognition by random order assembly and kinetic proofreading. $J$ Theor Biol 2007; 249:361-375.

29 Hess MT, Schwitter U, Petretta M, Giese B, Naegeli H. Bipartite substrate discrimination by human nucleotide excision repair. Proc Natl Acad Sci USA 1997; 94:6664-6669.

30 Patrick SM, Turchi John J. Stopped-flow kinetic analysis of replication protein A-binding DNA - Damage recognition and affinity for single-stranded DNA reveal differential contributions of k(on) and k(off) rate constants. J Biol Chem 2001; 276:2263022637.

31 Trego KS, Turchi J J. Pre-steady state binding of damaged DNA by XPC-RAD23B reveals a kinetic mechanism for damage discrimination. Biochemistry 2006; 45:1961-1969.

32 Tapias A, Auriol J, Forget D, et al. Ordered conformational changes in damaged DNA induced by nucleotide excision repair factors. J Biol Chem 2004; 279:19074-19083.

33 Hey T, Lipps G, Sugasawa K, Iwai S, Hanaoka F, Krauss G. The XPC-HR23B complex displays high affinity and specificity for damaged DNA in a true-equilibrium fluorescence assay. Biochemistry 2002; 41:6583-6587.

34 Sugasawa K, Okamoto T, Shimizu Y, Masutani C, Iwai S, Hanaoka F. A multistep damage recognition mechanism for global genomic nucleotide excision repair. Genes Dev 2001; 15:507-521.

35 Min JH., Pavletich NP. Recognition of DNA damage by the Rad4 nucleotide excision repair protein. Nature 2007; 449:570-575.

36 Truglio JJ, Karakas E, Rhau B, et al. Structural basis for DNA recognition and processing by UvrB. Nat Struct Mol Biol 2006; 13:360-364.

37 Mol CD, Arvai AS, Slupphaug G, et al. Crystal structure and mutational analysis of human uracil-DNA glycosylase: structural basis for specificity and catalysis. Cell 1995; 80:869-878.

38 Christine KS, MacFarlane AW, Yang K, Stanley RJ. Cyclobutylpyrimidine dimer base flipping by DNA photolyase. J Biol Chem 2002; 277:38339-38344.
39 Huang N, Banavali NK, MacKerell AD Jr. Protein-facilitated base flipping in DNA by cytosine-5-methyltransferase. Proc Natl Acad Sci USA 2003; 100:68-73.

40 Mees A, Klar T, Gnau P, Crystal structure of a photolyase bound to a CPD-like DNA lesion after in situ repair. Science 2004; 306:1789-1793.

41 Tubbs JL, Pegg AE, Tainer JA. DNA binding, nucleotide flipping, and the helix-turn-helix motif in base repair by O6-alkylguanine-DNA alkyltransferase and its implications for cancer chemotherapy. DNA Repair (Amst) 2007; 6:1100-1115.

42 Evans E, Fellows J, Coffer A, Wood RD. Open complex formation around a lesion during nucleotide excision repair provides a structure for cleavage by human XPG protein. EMBO J 1997; 16:625-638

43 Ikegami T, Kuraoka I, Saijo M, et al. Solution structure of the DNA- and RPA-binding domain of the human repair factor XPA. Nat Struct Biol 1998; 5:701-706.

44 Camenisch U, Dip R, Schumacher SB, Schuler B, Naegeli H. Recognition of helical kinks by xeroderma pigmentosum group $\mathrm{A}$ protein triggers DNA excision repair. Nat Struct Mol Biol 2006; 13:278-284.

45 Camenisch U, Dip R, Vitanescu M, Naegeli H. Xeroderma pigmentosum complementation group A protein is driven to nucleotide excision repair sites by the electrostatic potential of distorted DNA. DNA Repair (Amst) 2007; 6:1819-1828.

46 Hermanson-Miller IL, Turchi JJ. Strand-specific binding of RPA and XPA to damaged duplex DNA. Biochemistry 2002; 41:24022408.

47 Patrick SM, Turchi JJ. Xeroderma pigmentosum complementation group A protein (XPA) modulates RPA-DNA interactions via enhanced complex stability and inhibition of strand separation activity. J Biol Chem 2002; 277:16096-16101.

48 Patrick SM, Turchi JJ. Human replication protein A preferentially binds duplex DNA damaged with cisplatin. Biochemistry 1998; 37:8808-8815.

49 Treuner K, Ramsperger U, Knippers R. Replication protein A induces the unwinding of long double-stranded DNA regions. $J$ Mol Biol 1996; 259:104-112.

50 Patrick SM, Turchi JJ. Replication protein A (RPA) binding to duplex cisplatin-damaged DNA is mediated through the generation of single-stranded DNA. J Biol Chem 1999; 274:14972-14978.

51 Sugasawa K, Okuda Y, Saijo M, et al. UV-induced ubiquitylation of XPC protein mediated by UV-DDB-ubiquitin ligase complex. Cell 2005; 121:387-400.

52 Wang QE, Zhu QZ, Wani G, El-Mahdy MA, Li JY, Wani AA. DNA repair factor XPC is modified by SUMO-1 and ubiquitin following UV irradiation. Nucleic Acids Res 2005; 33:40234034.

53 Luijsterburg MS, Goedhart J, Moser J, et al. Dynamic in vivo interaction of DDB2 E3 ubiquitin ligase with UV-damaged DNA is independent of damage-recognition protein XPC. J Cell Sci 2007; 120:2706-2716.

54 Wu XM, Shell SM, Yang ZG, Zou Y. Phosphorylation of nucleotide excision repair factor xeroderma pigmentosum group a by ataxia telangiectasia mutated and Rad3-related-dependent checkpoint pathway promotes cell survival in response to UV irradiation. Cancer Res 2006; 66:2997-3005.

55 Ariza RR, Keyse SM, Moggs JG, Wood RD. Reversible protein phosphorylation modulates nucleotide excision repair of dam- 
aged DNA by human cell extracts. Nucleic Acids Res 1996; 24:433-440.

56 Patrick SM, Oakley GG, Dixon K, Turchi JJ. DNA damage induced hyperphosphorylation of replication protein A. 2. characterization of DNA binding activity, protein interactions, and activity in DNA replication and repair. Biochemistry 2005; 44:8438-8448.

57 Nuss JE, Patrick SM, Oakley GG, et al. DNA Damage induced hyperphosphorylation of replication protein A. 1. Identification of novel sites of phosphorylation in response to DNA damage. Biochemistry 2005; 44:8428-8437.

58 Oakley GG, Patrick SM, Yao JQ, Carty MP, Turchi JJ, Dixon K. RPA phosphorylation in mitosis alters DNA binding and proteinprotein interactions. Biochemistry 2003; 42:3255-3264.

59 Shen M, Berndt SI, Rothman N, et al. Polymorphisms in the DNA nucleotide excision repair genes and lung cancer risk in Xuan Wei, China. Int J Cancer 2005; 116:768-773.

60 Zienolddiny S, Campa D, Lind H, et al. Polymorphisms of DNA repair genes and risk of non-small cell lung cancer. Carcinogenesis 2006; 27:560-567.

61 Matakidou A, Eisen T, Fleischmann C, Bridle H, Houlston RS. Evaluation of xeroderma pigmentosum XPA, XPC, XPD, XPF, $\mathrm{XPB}, \mathrm{XPG}$ and DDB2 genes in familial early-onset lung cancer predisposition. Int J Cancer 2006; 119:964-967.

62 Vogel U, Overvad K, Wallin H, Tjonneland A, Nexo BA, Raaschou-Nielsen O. Combinations of polymorphisms in XPD, XPC and XPA in relation to risk of lung cancer. Cancer Lett 2005; 222:67-74.

63 Mechanic LE, Millikan RC, Player J, et al. Polymorphisms in nucleotide excision repair genes, smoking and breast cancer in African Americans and whites: a population-based case-control study. Carcinogenesis 2006; 27:1377-1385.

64 De Ruyck K, Szaumkessel M, De Rudder I, et al. Polymorphisms in base-excision repair and nucleotide-excision repair genes in relation to lung cancer risk. Mutat Res 2007; 631:101-110.

65 Clarkson SG, Wood RD. Polymorphisms in the human XPD (ERCC2) gene, DNA repair capacity and cancer susceptibility: an appraisal. DNA Repair (Amst) 2005; 4:1068-1074.

66 Manuguerra M, Saletta F, Karagas MR, et al. XRCC3 and XPD/ ERCC2 single nucleotide polymorphisms and the risk of cancer: a HuGE review. Am J Epidemiol 2006; 164:297-302.

$67 \mathrm{Au}$ WW. Heritable susceptibility factors for the development of cancer. J Radiat Res (Tokyo) 2006; 47(Suppl B):B13-B17.

68 Yang ZH, Liang WB, Jia J, Wei YS, Zhou B, Zhang L. The xeroderma pigmentosum group $\mathrm{C}$ gene polymorphisms and genetic susceptibility of nasopharyngeal carcinoma. Acta Oncol 2007; $1-6$.

69 Guo W, Zhou RM, Wan LL, et al. Polymorphisms of the DNA repair gene xeroderma pigmentosum groups $\mathrm{A}$ and $\mathrm{C}$ and risk of esophageal squamous cell carcinoma in a population of high incidence region of North China. J Cancer Res Clin Oncol 2008; 134:263-270.

70 Bai Y, Xu L, Yang X, et al. Sequence variations in DNA repair gene XPC is associated with lung cancer risk in a Chinese population: a case-control study. BMC Cancer 2007; 7:81.

71 Lee GY, Jang JS, Lee SY, et al. XPC polymorphisms and lung cancer risk. Int J Cancer 2005; 115:807-813.

72 Uchida A, Sugasawa K, Masutani C, et al. The carboxy-terminal domain of the XPC protein plays a crucial role in nucleotide excision repair through interactions with transcription factor IIH. DNA Repair 2002; 1:449-461.

73 Bartsch H, Dally H, Popanda O, Risch A, Schmezer P. Genetic risk profiles for cancer susceptibility and therapy response. Recent Results Cancer Res 2007; 174:19-36.

74 Popanda O, Schattenberg T, Phong CT, et al. Specific combinations of DNA repair gene variants and increased risk for nonsmall cell lung cancer. Carcinogenesis 2004; 25:2433-2441.

75 Einhorn LH. Curing metastatic testicular cancer. Proc Nat Acad Sci USA 2002; 99:4592-4595.

76 Muggia FM, Fojo T. Platinums: extending their therapeutic spectrum. J Chemother 2004; 16:77-82.

77 Popat S, O'Brien M. Chemotherapy strategies in the treatment of small cell lung cancer. Anti-Cancer Drugs 2005; 16:361-372.

78 Ozols RF, Bookman MA, Connolly DC, et al. Focus on epithelial ovarian cancer. Cancer Cell 2004; 5:19-24.

79 Hanna NH, Einhorn LH. Small-cell lung cancer: state of the art. Clin Lung Cancer 2002; 4:87-94.

80 Einhorn LH. Curing metastatic testicular cancer. Proc Natl Acad Sci USA 2002; 99:4592-4595.

81 Koberle B, Masters JRW, Hartley JA, Wood RD. Defective repair of cisplatin-induced DNA damage caused by reduced XPA protein in testicular germ cell tumours. Current Biology 1999; 9:273-276.

82 Welsh C, Day R, McGurk C, Masters JRW, Wood RD, Koberle B. Reduced levels of XPA, ERCC1 and XPF DNA repair proteins in testis tumor cell lines. Int J Cancer 2004; 110:352-361.

83 Spitz MR, Wei QY, Dong Q, Amos CI, Wu XF. Genetic susceptibility to lung cancer: the role of DNA damage and repair. Cancer Epidemiol Biomarkers Prev 2003; 12:689-698.

84 Kartalou M, Essigmann JM. Mechanisms of resistance to cisplatin. Mutat ResFund Mol Mech Mutagen 2001;478:23-43.

85 Andrews BJ, Turchi JJ. Development of a high-throughput screen for inhibitors of replication protein $\mathrm{A}$ and its role in nucleotide excision repair. Mol Cancer Ther 2004; 3:385-391.

86 Niedernhofer LJ, Garinis GA, Raams A, et al. A new progeroid syndrome reveals that genotoxic stress suppresses the somatotroph axis. Nature 2006; 444:1038-1043.

87 Dolle ME, Busuttil RA, Garcia AM, et al. Increased genomic instability is not a prerequisite for shortened lifespan in DNA repair deficient mice. Mutat Res 2006; 596:22-35. 